\title{
Externality reductions in residential areas due to rail transit networks
}

\author{
Chaug-Ing Hsu' ${ }^{1}$, Shwu-Ping Guo ${ }^{2}$ \\ ${ }^{1}$ Department of Transportation Technology and Management, National Chiao Tung University, \\ Hsinchu, 300, Taiwan, Republic of China (e-mail: cihsu@cc.nctu.edu.tw) \\ ${ }^{2}$ Department of Logistics Management, Leader University, Tainan, 709, Taiwan, Republic of \\ China (e-mail: spguo@mail.leader.edu.tw)
}

Received: February 2004 / Accepted: September 2004

\begin{abstract}
This work adopts a continuum approximation method based on long-term planning to describe the continuous characteristics of air pollution dispersion, street and rail transit network flow as well as household distribution. The model of air pollution distribution in a metropolitan area with surface streets and rail transit networks is constructed by applying dispersion equations of air pollutants in the field of Physics. Variations in air pollution due to decreased car flows, which result from commuters' shifting to the newly completed rail transit system, are also analyzed. Benefits from the reduction of air pollution are estimated by accumulating all of the exposed households at each residential site. Finally, a case study demonstrates the model application and estimates the improvement of residential environment in the Taipei metropolitan area due to the rail transit system.
\end{abstract}

JEL classification: R40, R41

\section{Introduction}

Economic growth and industrial activities stimulate urban passenger travel. Transportation supply, which stimulates land use and urban development, is expanded by increasing travel demand. Alternatively, it creates air pollution as well as traffic congestion. Excess urban development negatively impacts environmental quality as well. This issue has drawn intensive public attention and caused authorities to synchronize transportation system development, urban growth and environment quality by enforcing environmental protection driven by urban planning and land-use regulations. Conventional transportation strategies include enhancing public transportation, creating automobile prohibited zones, prohibiting high-pollution fuels, implementing high occupancy vehicle lanes, car pools and parking regulations. Land-use

The authors would like to thank the National Science Council of the Republic of China for financially supporting this research under Contract No. NSC89-2211-E-009-077. The constructive comments of the editor, Professor Roger R. Stough, are greatly appreciated. 
strategies include land-use zoning, cubic content regulations and increased open spaces. Previous studies have developed integrated transportation and environmental system models to understand their interactions (e.g., Scott et al., 1997; Bielli et al., 1998; Gualtieri and Tartaglia 1998; Ambrosino et al., 1999). In addition to failing to formulate models to understand how urban household spatial distribution and mode choices, air pollution and congestion are related, the above models did not estimate transportation externalities based on these relationships.

Transportation planning and subsequent environmental impacts are inseparable issues. Relevant studies have constructed sequential models to examine the environmental impacts of transportation systems on ecology and energy consumption. Recent studies applied dispersion equations of particles to portray pollutant diffusion. Furthermore, they estimated the distributions and calibrated relevant parameters within different conditions (e.g., Prabha and Radlgruber 1999). Alternatively, to design pollution-proof measures, studies involving transportation externalities and environmental impacts mainly used field surveys to estimate aggregated air pollution for different traffic zones. However, air pollution dispersion and household spatial distributions are continuous. Heterogeneity of air pollution distribution at various residential sites that have different numbers of households and car flows is neglected when the traffic zoning method is adopted. Additionally, perceived external cost of air pollution varies with the number of exposed households at each residential site. Restated, although air pollutants at two different residential sites are identical, their total perceived external costs differ from the perspective of community or society if the total number of exposed households is not identical.

Therefore, this work adopts a continuum approximation method to construct the model of air pollution distribution and to estimate the perceived external cost in terms of the number of exposed households at each residential site. The continuous characteristics of air pollution dispersion, street and rail transit network flow as well as household distribution are characterized to formulate how car flows and distribution of air pollution are related at each residential site. The model of air pollution in a metropolitan area with surface streets and rail transit networks is constructed by applying dispersion equation of air pollutants in the field of Physics. Hsu and Guo (2001) developed a household mode choice and residential distribution model. This paper adopts a modification of the model to analyze variations in air pollution at each residential site due to decreased traffic flows that result from commuters' shifting to the newly completed rail transit system. Moreover, the study further estimate benefits from air pollution reduction due to the completion of rail transit networks by accumulating all of the exposed households at each residential site and then aggregating households' external cost reduction. The unique contribution of the paper is to develop a new model, which augments the model of Hsu and Guo (2001) with the dispersion equation of air pollutants in the field of Physics. The developed model estimates variations in pollution at each residential site and aggregates this up to total estimates while relating air pollution distribution with household spatial distribution, mode choices, and traffic congestion. The bottom-up estimation procedure considers continuous property and heterogeneity of air pollution distribution at various residential sites with different number of households and car flows and thereby reduce the aggregation bias. 
The model of air pollution distribution developed in this study considers factors such as the emission rate per automobile, car flow, wind speed, wind direction and distance between the pollution source and receptor. First, a dispersion function of air pollution is formulated to illuminate the amount of pollutants generated from automobiles at each residential site. An air pollution distribution model is then proposed to indicate the pollutant concentration at each residential site. Moreover, experimental results of the model are further calculated to analyze benefits from air pollution reduction in terms of the number of exposed households at different residential sites following the completion of rail transit networks.

\section{Air pollution distribution model}

Providing effective transportation services depends on sufficient energy consumption to generate motion power so that travelers can move from A to B promptly and efficiently. However, when energy consumption generates motion power, by-products such as $\mathrm{CO}, \mathrm{HC}, \mathrm{TSP}, \mathrm{NO}_{\mathrm{x}}$, and $\mathrm{SO}_{\mathrm{x}}$, which impair human health are also generated. Comparisons of air pollution among urban modes indicate that automobiles and buses, which are fueled by gasoline, are more polluting than other modes are. Owing to the increase in environmental protectionism and emphasis on external costs, transportation costs should not only include personal travel time, out-of-packet cost but also total social and external costs. Air pollutants disperse downwind areas via air and wind. Namely, air pollution is distributed continuously throughout the study area rather than restricted to generated sites. Therefore, this work applies the dispersion equation of air pollution to formulate related models. Experimental results of the households' residential distribution model and households' mode choices and residential distribution model as constructed by Hsu and Guo (2001) are further applied to analyze the operating benefits of rail transit networks. To elucidate the continuous property of car flows and air pollution, this work follows the studies of Hsu and Guo (2001) and Yang et al. (1994) and assumes that surface streets in the study area are continuous and homogeneous networks, which are represented by a two-dimensional coordinate system $D$. Where $\partial D$ denotes the boundary of the study area.

\subsection{Dispersion and distribution of air pollution}

This work refers to the Gaussian model of air pollutant concentration, which describes the three dimensional concentration field generated by a point polluting source under stationary meteorological and emission conditions (e.g., Zannetti 1990; de Nevers 1995). Let three-dimensional coordinate $s=\left(s_{1}, s_{2}, s_{3}\right)$ represent a residential site, which generates air pollution. When air pollution is generated from residential site $s=\left(s_{1}, s_{2}, s_{3}\right)$, the concentration of air pollution $a p(s, r)$ at downwind residential site $r=\left(r_{1}, r_{2}, r_{3}\right)$, which receives air pollution, is formulated as Eq. (1): 


$$
a p(s, r)=\frac{Q}{2 \pi \sigma_{h} \sigma_{z}|\bar{w}|} \exp \left[-\frac{1}{2}\left(\frac{\Delta_{c w}}{\sigma_{h}}\right)^{2}\right] \cdot \exp \left[-\frac{1}{2}\left(\frac{h_{e}-r_{3}}{\sigma_{z}}\right)^{2}\right]
$$

Where $Q$ denotes the emission rate per automobile, and $\sigma_{h}$ and $\sigma_{z}$ represent the standard horizontal and vertical deviations of the concentration distribution, respectively. Additionally, $\bar{w}$ denotes the average wind velocity vector $\left(\bar{w}_{1}, \bar{w}_{2}, \bar{w}_{3}\right) . \Delta_{c w}=\sqrt{|r-s|^{2}-d^{2}}$ is the crosswind distance from air pollution source $s=\left(s_{1}, s_{2}, s_{3}\right)$ to receptor $r=\left(r_{1}, r_{2}, r_{3}\right) \cdot d=\frac{(r-s) \cdot \bar{w}}{|\bar{w}|}$ is the downwind distance from the air pollution source $s=\left(s_{1}, s_{2}, s_{3}\right)$ to receptor $r=\left(r_{1}, r_{2}, r_{3}\right)$. Where $h_{e}$ is the effective emission height of air pollution. Green et al. (1980) present $\sigma_{h}(d)$ and $\sigma_{z}(d)$ in an analytical form as Eq. (2) where parameters $k_{1}$, $k_{2}, k_{3}, k_{4}$, and $k_{5}$ are summarized in Table 1 .

$$
\sigma_{h}(d)=\frac{k_{1} \cdot d}{\left[1+\left(d / k_{2}\right)\right]^{k_{3}}}, \quad \sigma_{z}(d)=\frac{k_{4} \cdot d}{\left[1+\left(d / k_{2}\right)\right]^{k_{5}}}
$$

An air pollution distribution equation is formulated to estimate the air pollution reduction in the study area owing to a portion of the commuters, who originally traveled by automobile, have shifted to a rail transit system after its completion. Estimated benefits of air pollution reduction are measured by analyzing the social benefits of exposed households' health improvement and decrease in mortality following pollution reduction. The Gaussian model of air pollution concentration is converted into a spatial distribution model in a twodimensional surface. Namely, the concentration of air pollution from all residential sites that disperses to each specific residential site and is in the vertical range where pollutant will affect human health are aggregated. Regarding air pollution sources, this work merely considers car flows on surface streets and assumes that the emission rate per car is identical. A static model of air pollution distribution is developed; therefore, average wind direction and average wind velocity are treated as atmospheric parameters. Moreover, atmospheric parameters affect the dispersion pattern of air pollution at each residential site. Car emissions within the same residential site generate identical dispersion patterns. Aggregated air pollution at each residential site depends on car flows within that site, but also on the air pollution concentration that is dispersed

Table 1. Values of parameters $k_{1}, k_{2}, k_{3}, k_{4}$ and $k_{5}$

\begin{tabular}{llllllr}
\hline Stability classification & Pasquill categories & $k_{1}$ & $k_{2}$ & $k_{3}$ & $k_{4}$ & $k_{5}$ \\
\hline Extremely unstable & A & 0.2500 & 927 & 0.189 & 0.1020 & -1.918 \\
Moderately unstable & B & 0.2020 & 370 & 0.162 & 0.0962 & -1.101 \\
Slightly unstable & C & 0.1340 & 283 & 0.134 & 0.0722 & 0.102 \\
Neutral & D & 0.0787 & 707 & 0.135 & 0.0475 & 0.465 \\
Slightly stable & E & 0.0566 & 1070 & 0.137 & 0.0335 & 0.624 \\
Moderately stable & F & 0.0370 & 1170 & 0.134 & 0.0220 & 0.700 \\
Extremely stable & G & - & - & - & - & - \\
\hline
\end{tabular}


from other residential sites. Therefore, the air pollution distribution at residential site $x=\left(x_{1}, x_{2}\right)$ can be formulated as Eq. (3):

$$
\begin{aligned}
& \operatorname{ad}\left(x_{1}, x_{2}\right) \\
& =\iint_{D}\left|v\left(z_{1}, z_{2}\right)\right| \cdot\left[\int_{0}^{R} a p(z, x) d z_{3}\right] d z_{1} d z_{2} \\
& =\iint_{D} \frac{\left|v\left(z_{1}, z_{2}\right)\right| \cdot Q}{2 \pi}\left\{\int_{R 1}^{R 2} \frac{1}{\sigma_{h} \cdot \sigma_{z} \cdot|\bar{w}|} \exp \left[-\frac{1}{2}\left(\frac{|\bar{w}|^{2} \cdot|x-z|^{2}-((x-z) \cdot \bar{w})^{2}}{|\bar{w}|^{2} \cdot \sigma_{h}^{2}}\right)\right] .\right. \\
& \left.\times \exp \left[-\frac{1}{2}\left(\frac{h_{e}-z_{3}}{\sigma_{z}}\right)^{2}\right] d z_{3}\right\} d z_{1} d z_{2}
\end{aligned}
$$

Where $\left|v\left(s_{1}, s_{2}\right)\right|$ denotes car flows from residential site $s=\left(s_{1}, s_{2}\right)$ to the CBD and is one of the model results in Hsu and Guo (2001). Where $[R 1, R 2]$ denotes the vertical range in which pollutants become detrimental to human health.

\subsection{Revised model}

The above mentioned models are formulated in a continuous space and their decision variables are dependent on the locations of residential sites, which are represented by a two-dimensional coordinate system and difficult to solve. Thus, the finite element method is applied herein to dissever domains of air pollution and noise distribution models into a finite number of triangular elements (sub-domain) with vertexes that represent residential sites. Let $e(\forall e \in E)$ represent a triangular element where $E$ is the set of elements. Let $e_{i}$, $e_{j}$ and $e_{k}$ denote the three vertexes of a triangular element, respectively. Subsequently, the set of vertexes of all triangular elements in the study area is formulated as $B=\cup_{e \in E}\left(e_{i} \cup e_{j} \cup e_{k}\right)$. Vertexes of all triangular elements are considered herein $\underset{a s}{e \in E}$ residential sites and synchronize coordinates of residential sites with those of element vertexes. Namely, each coordinate of element vertex, $b=\left(b_{1}, b_{2}\right)(\forall b \in B)$, represents a residential site to simplify the solving procedure of air pollution distribution model. The discrete function of air pollution distribution at residential site $x=\left(x_{1}, x_{2}\right)$ is formulated as Eq. (4).

$$
\begin{aligned}
& \operatorname{ad}\left(x_{1}, x_{2}\right) \\
& =\sum_{b \in B} \frac{|v(b)| \cdot Q}{2 \pi \cdot}\left\{\sum_{[R 1, R 2]} \frac{1}{\sigma_{h} \cdot \sigma_{z} \cdot|\bar{w}|} \exp \left[-\frac{1}{2}\left(\frac{|\bar{w}|^{2} \cdot|x-b|^{2}-((x-b) \cdot \bar{w})^{2}}{|\bar{w}|^{2} \cdot \sigma_{h}^{2}}\right)\right]\right. \\
& \left.\times \exp \left[-\frac{1}{2}\left(\frac{h_{e}-b_{3}}{\sigma_{z}}\right)^{2}\right]\right\}
\end{aligned}
$$




\section{Benefit analysis}

Experimental results of the air pollution distribution model (Sect. 2) are previews for variations in cumulative air pollution before and after the rail transit networks are completed. Implementation of rail transit networks can alter travelers' mode choices and car flows, and then reduces cumulative volumes of air pollution in some residential sites. Previous studies have concluded that transportation externalities of air pollution undermine human health (e.g., Alberini et al., 1997; Aunan et al., 1998). Otterstrom (1995) estimated social costs of air pollution in terms of damage such as diseases, crop yield loss and comfort and further priced their damage. A parameter, $\theta_{1}$, that represents social cost per unit air pollution is selected to estimate the benefit of its reduction following the completion of rail transit networks. The benefit of reducing air pollution at residential site $x=\left(x_{1}, x_{2}\right), R B\left(x_{1}, x_{2}\right)$, is formulated as Eq. (5).

$$
\begin{aligned}
& R B\left(x_{1}, x_{2}\right) \\
& =\theta_{1} \cdot\left[\sum_{i \in I} g^{i, B E F}\left(x_{1}, x_{2}\right) \cdot a d^{B E F}\left(x_{1}, x_{2}\right)-\sum_{i \in I} g^{i, A F T}\left(x_{1}, x_{2}\right) \cdot a d^{A F T}\left(x_{1}, x_{2}\right)\right]
\end{aligned}
$$

Where BEF and AFT denote before and after the rail transit networks are completed, respectively. Hence, $g^{i, B E F}\left(x_{1}, x_{2}\right)$ and $a d^{B E F}\left(x_{1}, x_{2}\right)$ represent the number of $i$ th income level households and air pollution, respectively, at residential site $x=\left(x_{1}, x_{2}\right)$ before the rail transit networks are completed. While $g^{i, A F T}\left(x_{1}, x_{2}\right)$ and $\operatorname{ad}^{A F T}\left(x_{1}, x_{2}\right)$, respectively, represent those variables after the rail transit networks are completed.

\section{Illustrative example}

To illustrate the application and experimental results, the models described above as well as the models in Hsu and Guo (2001) are applied to the Taipei metropolitan area and the Taipei Rapid Transit System (Fig. 1). Table 2 summarizes the parameter values of air pollution distribution model.

Figures $2 a$ and $2 b$ illustrate distributions of cumulative air pollution before and after the completion of rail transit networks, respectively. Prior to completion, the spatial distribution of cumulative air pollution displays significant peaking characteristics (Fig. 2a). Residential sites with higher cumulative air pollution are clustered around areas near CBD such as Ta-An, Chung-Jeng, Shin-Yi and Chung-Shan in Taipei City. This finding also implies that the distribution of cumulative air pollution is highly interrelated with locational developments and surface street networks in a metropolitan when rail transit networks are not incorporated. Alternately, the spatial convergence of cumulative air pollution distribution is moderated due to the completion of rail transit networks (Fig. 2b). The difference of cumulative air pollution between areas surrounding CBD and outlying areas of a metropolitan is diminished. While residential sites with higher cumulative air pollution are clustered around outlying areas such as Mu-Cha and Shih-Lin areas in Taipei City as well as Chung-Ho, Yong-Her, San-Chong areas in Taipei County. This finding implies that the completion of rail transit 
networks results in a variation in mobility at each residential site. Therefore, more households are willing to live in outlying areas of a metropolitan. Cumulative air pollution is gradually and smoothly distributed throughout the study area and spatial distribution peaks of cumulative air pollution are also moderated due to a decrease in car flows surrounding the CBD and an increase in car flows that are generating around outlying areas of a metropolitan.

Figure 3 illustrates the equi-variation contours of cumulative air pollution in the study area after the rail transit networks are completed. The cumulative air pollution in areas surrounding CBD such as Ta-Tung, Chung-Shan, SungShan in Taipei City decreases, while in outlying areas such as $\mathrm{Mu}-\mathrm{Cha}$ in Taipei City and Chung-Ho, Yong-Her in Taipei County it increases. Notably, the cumulative air pollution in Nei-Hu, which is in outlying areas of Taipei City, decreases rather than increases. This situation may be owing to that some CBD-oriented car flows divert to rail transit stations in the outlying areas of Taipei City and then transfer to rail transit rather than proceed to the CBD by car.

Based on the method of Aunan et al. (1998), this work estimates that the benefits of $1,000 \mathrm{~g}$ reduction of cumulative air pollution perceived by one household per day $\theta_{1}$ is equal to US\$ $1.834 \times 10^{-5}$. From Eq. (5) and cumulative air pollution, respectively, benefits from daily air pollution reduction in terms of exposed households after the completion of rail transit networks can be estimated (Table 3). Table 3 reveals that if we aggregate the operating benefits of rail transit networks from air pollution reductions in terms of the number of exposed households, that is, the value of $\sum$ (the number of households at each residential site before the completion of rail transit networks $\times$ the cumulative air pollution at that residential site) $-\sum$ (the number of households at each residential site after the completion of rail transit networks $\times$ the cumulative air pollution at that residential site) multiplies the value of parameter $\theta_{1}$, then total benefit of air pollution reductions is US 736.933/day. The aggregated operating benefits of rail transit networks are tremendous if they are analyzed based on annual costs. Moreover, quantifying external effects of transportation systems on humans in terms of the number of exposed households is more beneficial to authorities not only to evaluate the feasibility, but also to estimate the aggregated benefit of a new transportation system.

\section{Conclusions}

The contribution of the paper is to develop an integrated model, which augments the model of Hsu and Guo (2001) with the dispersion equation of

Table 2. Parameter values of air pollution distribution model

\begin{tabular}{lll}
\hline Symbol & Definition & Value \\
\hline$Q$ & $\begin{array}{l}\text { Emission rate } \\
\text { per automobile } \\
\left.\text { (including CO, HC, TSP, and } \mathrm{NO}_{\mathrm{x}}\right) .\end{array}$ & $17.7 \mathrm{~g} / \mathrm{km}$ \\
Average wind velocity & 60 meters $/ \mathrm{min} \sim 360 \mathrm{~meters} / \mathrm{min}$ \\
\hline
\end{tabular}




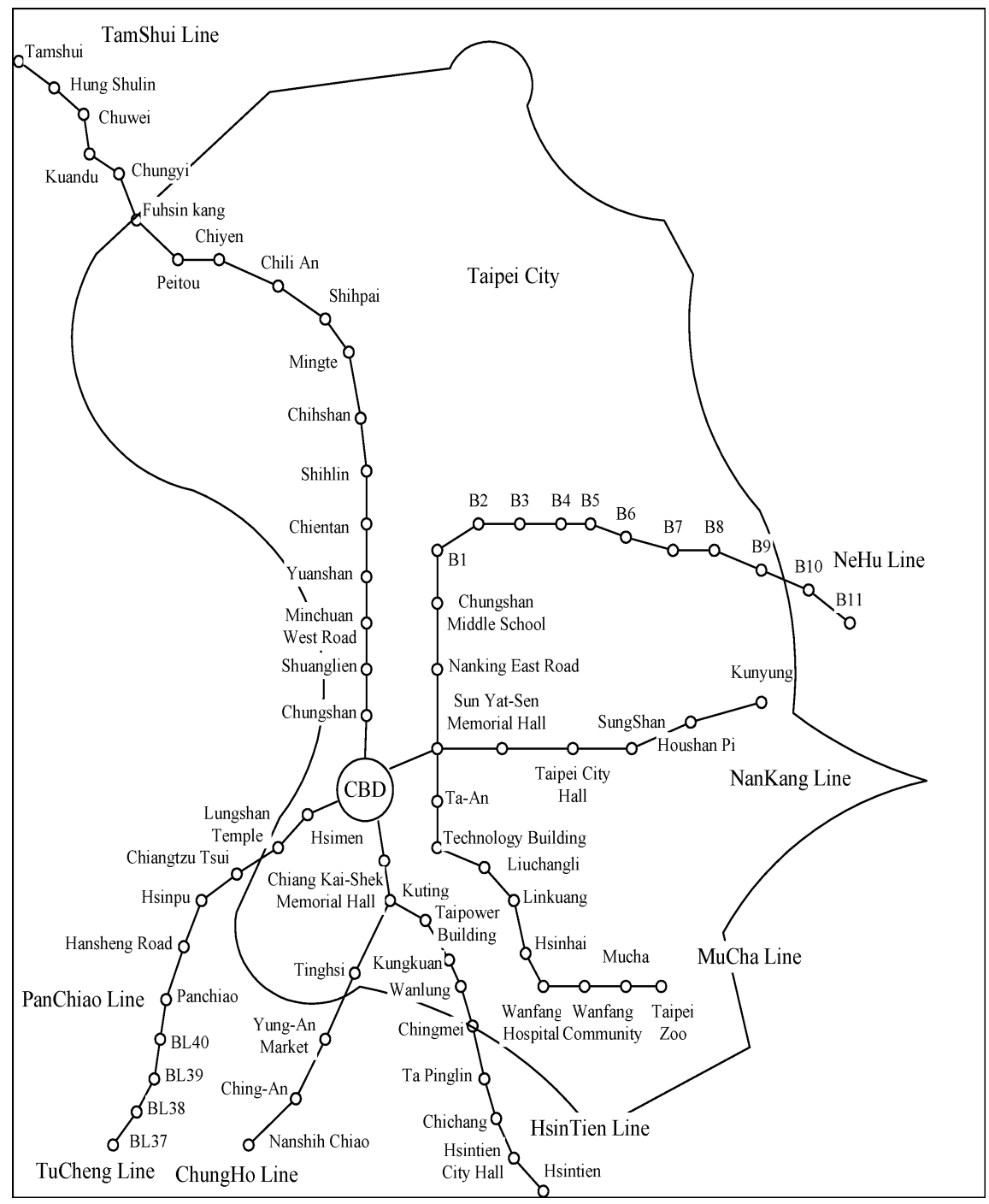

Fig. 1. The diagram of study area

air pollutants in the field of Physics. The developed model estimates variations in pollution at each residential site due to decreased traffic flows that result from commuters' shifting to the newly completed rail transit system and aggregates this up to total estimates. The bottom-up estimation procedure considers continuous property and heterogeneity of air pollution distribution at various residential sites with different number of households and car flows and thereby reduce the aggregation bias.

The illustrative example employed herein reveals the following results. First, the spatial convergence of cumulative air pollution distribution is 
a

without rail transit network
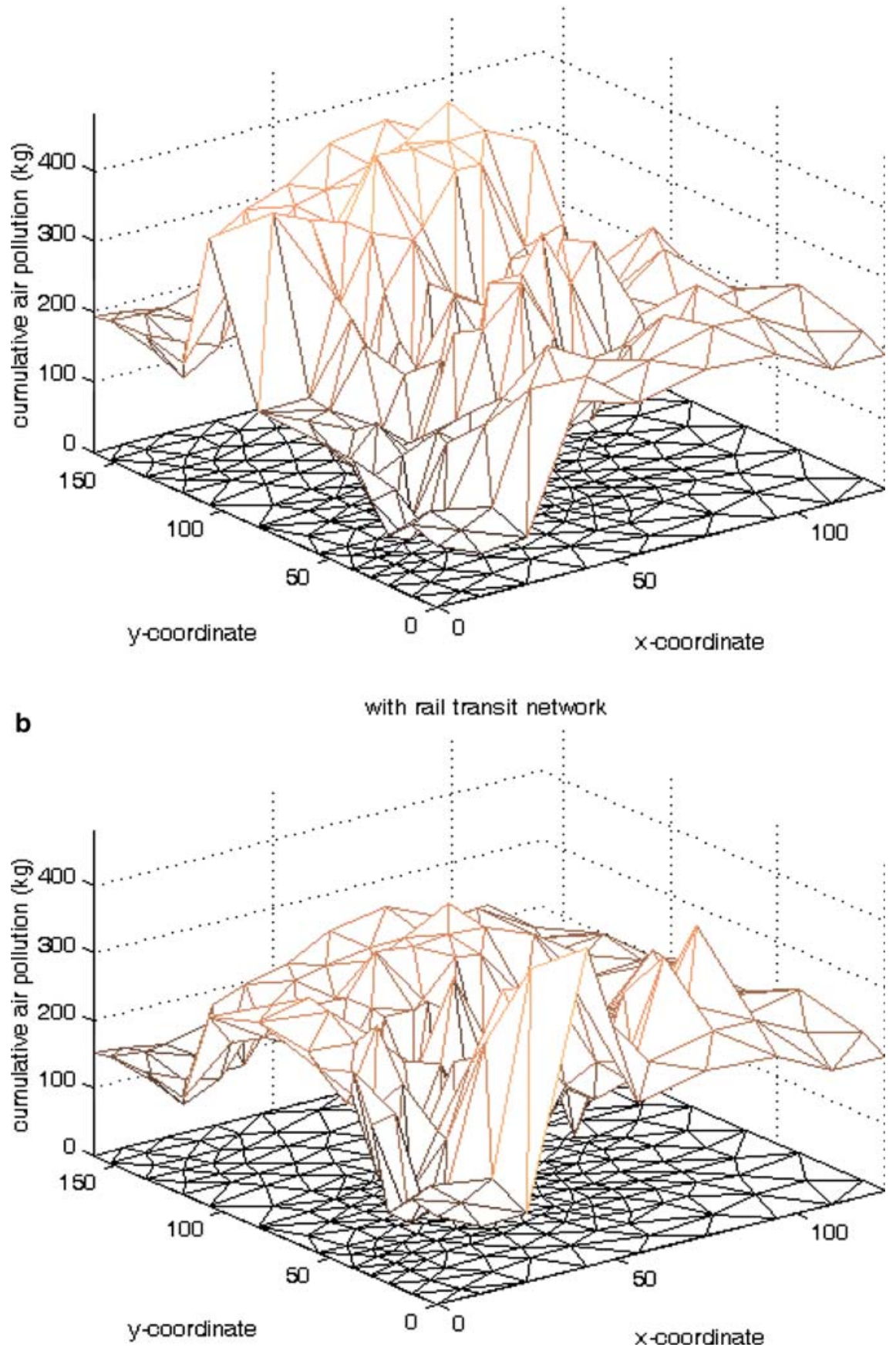

Fig. 2a. The cumulative air pollution at each residential site before the completion of rail transit networks. b The cumulative air pollution at each residential site after the completion of rail transit networks 


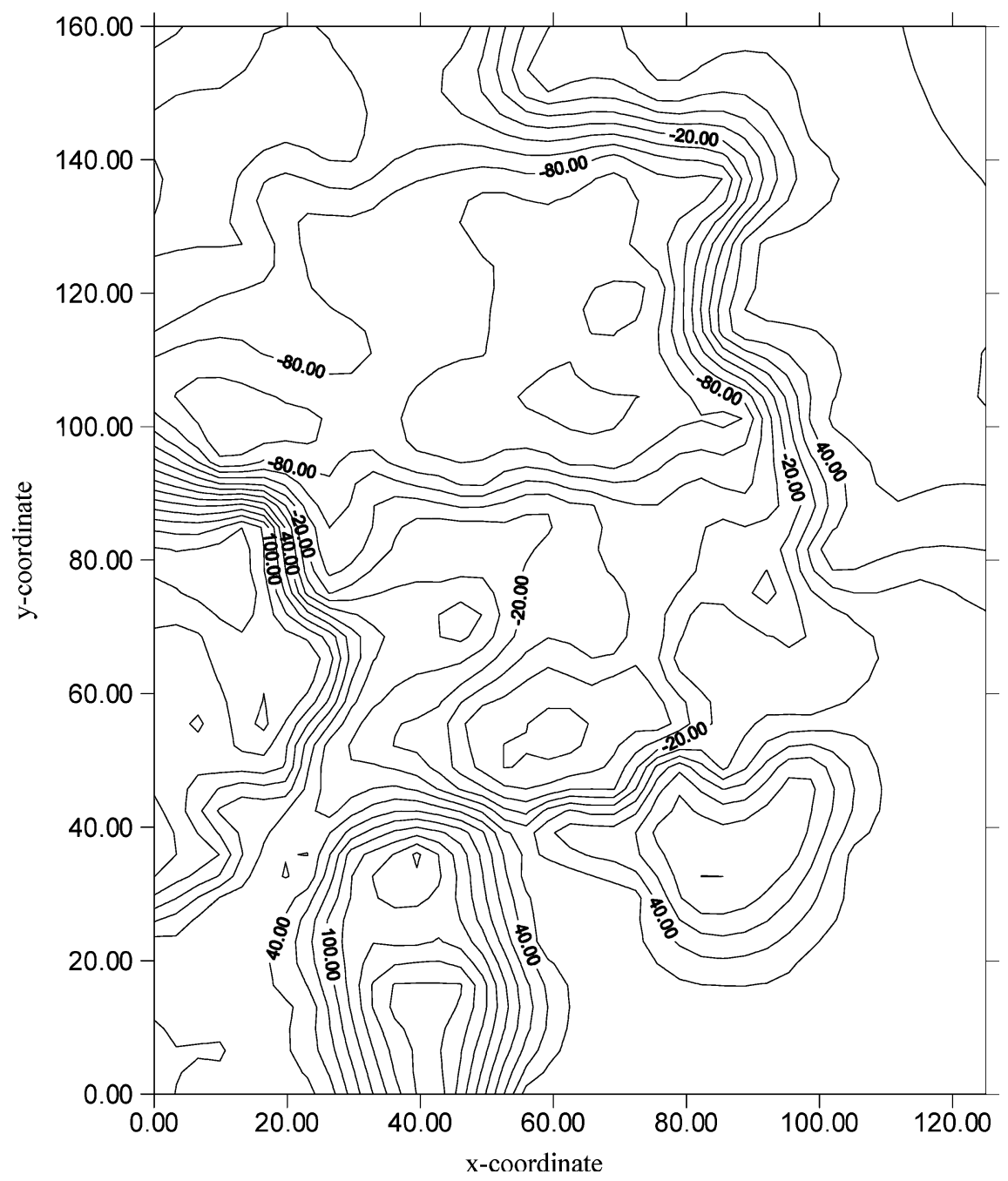

Fig. 3. Equi-variation contours of cumulative air pollution in the study area after the completion of rail transit networks. (unit: $\mathrm{kg}$ )

moderated. Hence, differences of air pollution between areas around CBD and outlying areas within the study area are diminished after rail transit networks are completed. Second, quantifying external effects of transportation systems on humans in terms of the number of exposed households is

Table 3. Benefits from air pollution reduction

\begin{tabular}{llll}
\hline & $\begin{array}{l}\text { Before pollution } \times \text { the } \\
\text { number of households }\end{array}$ & $\begin{array}{l}\text { After pollution } \times \text { the } \\
\text { number of households }\end{array}$ & Benefits (US\$/day) \\
\hline Air pollution & 654482.489 (unit: $1,000 \mathrm{~g})$ & 614300.737 (unit: $1,000 \mathrm{~g})$ & 736.933 \\
\hline
\end{tabular}


more beneficial to authorities not only to evaluate the feasibility but also to estimate the aggregated benefit of a new transportation system. Finally, distributions of cumulative air pollution before and after the completion of rail transit networks presented herein are referential in determining abatement strategies of air pollution and air pollution taxes.

This work analyzes the distributions of cumulative air pollution in a monocentric metropolitan area where commuters are assumed to drive directly to the $\mathrm{CBD}$ via surface streets or park and ride rail transit lines to the CBD. A static distribution model of air pollution is illustrated in terms of cumulative volume during the study period. However, the number of work trips varies with time. The departure time of households is dependent on the distance between residential site and the CBD, traffic conditions and the beginning of work. Therefore, the number and the time of work trip generations are attributed to dynamic probability distributions. Future studies should investigate the time-dependent distributions of households' trip generations, calibrate relevant parameters and construct instantaneously distribution model of air pollution so as to understand the relation between environmental impacts and traveler behaviors. On the other hand, the assumption of monocentric metropolitan is unrealistic because many urban areas of the world have become polycentric cities. Future studies should extend the developed model using a many-to-many demand pattern to explore the reductions of air pollutants at each residential site due to the completion of rail transit networks in a polycentric urban.

\section{References}

Alberini A, Cropper M, Fu TT, Krupnick A, Liu JT, Shaw DG, Harrington W (1997) Valuing health effects of air pollution in developing countries: The case of Taiwan. Journal of Environmental Economics and Management 34:107-126

Ambrosino G, Sassoli P, Bielli M, Carotenuto P, Romanazzo M (1999) A modeling framework for impact assessment of urban transport systems. Transportation Research 4D:73-79

Aunan K, Patzay G, Aaheim HA, Seip HM (1998) Health and environmental benefits from air pollution reductions in Hungary. The Science of the Total Environment 212:245-268

Bielli M, Carotenuto P, Site VD (1998) Transport and environment interactions: The Italian framework. Transportation Research 3D:389-397

Chen SW, Liu KT, Chang P, Ni PJ, Lin YM (1997) Estimations of vehicle air pollution emissions and relevant control strategies in Taiwan area. Journal of Environment Engineer 8:75-120 (In Chinese)

De Nevers N (1995) Air pollution control engineering. McGraw-Hill, New York

Green AE, Singhal RP, Venkateswar R (1980) Analytic extensions of the Gaussian plume model. JAPCA 30: 773-776

Gualtieri G, Tartaglia M (1998) Predicting urban traffic air pollution: A GIS framework. Transportation Research 3D:329-336

Hsu CI, Guo SP (2001) Household-mode choice and residential-rent distribution in a metropolitan area with surface road and rail transit networks. Environment and Planning 33A: $1547-1575$

Otterstrom T (1995) Pricing of environmental impacts of emissions from road traffic - Recent and present research in Finland. The Science of the Total Environment 169:311-319

Prabha TV, Radlgruber EM (1999) Investigation of air pollution distribution in Linz: Case studies to evaluate a K-type diffusion model coupled with a mass-consistent wind model. Atmospheric Environment 33:4067-4080

Scott DM, Kanaroglou PS, Anderson WP (1997) Impacts of commuting efficiency on congestion and emissions: Case of the Hamilton CMA, Canada. Transportation Research 2D:245-257 
Yang H, Yagar S, Iida Y (1994) Traffic assignment in a congested discrete/continuous transportation system. Transportation Research 28B: 161-174

Zannetti P (1990) Air pollution modeling-theories, computational methods and available software. Computational Mechanics Publications, New York 